\title{
MSIS IMAGE POSTIONING IN PORT AREAS WITH THE AID OF COMPARATIVE NAVIGATION METHODS
}

\author{
Natalia Wawrzyniak \\ Maritime University of Szczecin, Poland \\ Andrzej Stateczny \\ Marine Technology Ltd., Poland
}

\begin{abstract}
The article presents a method to determine the position of mechanically scanned sonar images by comparing them with the database of simulated synthetic images. The synthetic images are generated from high-density bathymetric data coming from the same fragment of water region, using the ray tracing method. The article discusses the issues related to the choice of the probability function as the method of image comparing which allows to find the correct georeference of the real image. For the correlation method and the logical conjunction method, which are believed to give the best results, detailed studies were performed, including boundary cases. The obtained results of matching are presented in tabular and graphic form.
\end{abstract}

Keywords: sonar imaging, MSIS sonar, image comparison methods, image simulation

\section{INTRODUCTION}

Mechanically Scanned Imaging Sonars, MSIS, are most frequently used for bottom searching in water regions of certain navigational interest, for the purpose of building hydraulic constructions or stocktaking of port areas and quays [1]. These sonars are specific for high resolution of the recorded images and relatively small spatial range. The MSIS images of port areas are full of characteristic objects or distinctive bottom fragments [2]. In a stationary variant, the sonar is equipped with a high-frequency converter which allows it to detect objects with the cubic capacity of some tens of cubic centimetres. Of certain interest are the attempts to use MSISs to track moving underwater objects [3]. The applied technology of converting the acoustic signal echo into the line of pixels remains the same as for side scan sonars, SSS. However, due to its independence from the surface research vessel, which eliminates problems with its motion, and the use of high-frequency converter, the MSIS sonar provides real opportunity to visualise underwater areas in a way close to that offered by photography. Unfortunately, the mode of independent operation on the seabed, without rigid connection with the surface vessel or even without any relation with it, makes establishing a precise position of the image recorded by MSIS a problem. A number of underwater positioning systems are being widely used now, but their high cost and time consuming operation of setting them up make the use of these systems unreasonable for the abovementioned tasks. The issues of underwater positioning are discussed in [4-6], among other sources.

The article proposes to use comparative navigation methods for establishing positions of stationary MSIS images with the aid of the model of seabed and the synthetic sonar image simulator. It is assumed that precise and up-to-date bathymetric data for the port area of interest are available and that these data will allow to generate the database of synthetic images with the resolution close to that of the original images. 
The article discusses the issues of MSIS imaging and generation of their artificial seabed model based equivalents for the purposes of MSIS positioning. The presented method of sonar image positioning bases on comparing the real image with the database of the generated synthetic images. The flowchart of this process is included. In the performed simulation experiment, different probability functions are compared to find the function which returns best MSIS image matching.

\section{BACKGROUND}

\section{MSIS IMAGING OF PORT AREAS}

The operating principle of imaging sonars bases on echo energy measurements of the acoustic wave emitted, per unit time, by the converter and reflected from seabed elements. Series of amplitudes of successive pulses are recorded as lines of pixels and, after geometry correction and amplification, can be presented as a single-channel image of the seabed. The stationary MSIS performs mechanical scanning in horizontal plane by rotating the converter. During the scanning process, the scanning head rotates step by step by a given constant angle $\varepsilon$, each time emitting the beam of acoustic waves and recording the reflected echo. The emitted acoustic wave has the form of a fan, narrow in the horizontal plane and wide in the vertical plane. The main maximum axis of the wave directionality characteristic is directed downwards of the horizon line, by ten to twenty degrees, as a result of which most of the acoustic energy is directed towards the seabed. The record of the imaging signal having the form of successive lines of pixels can be presented in the way similar to that used in side scan sonars (Fig. 1a); with the only difference that in MSIS imaging all sonar lines have common origin at the sonar head position. This origin is simultaneously the centre of the image, and when we know the head rotation angle $\varepsilon$ we can determine relative positions of particular lines with respect to each other. This Cartesian mapping of the image is very convenient from the point of view of photo interpretation (Fig.1b). Image correction is not necessary, except pixels situated very close to the converter and being part of the so-called dead zone of the sonar image. The orientation, with respect to North, of the image recorded by the scanning sonar is obtained from the compass integrated with the sonar head. If certain objects with known locations (part of quay, bridge pillar) or those which provide an opportunity to be precisely localised (mooring post) are recorded in the image, they can be used as markers to calculate the position of the receiver, based on the known range of the beam. However, this is not a universal solution.
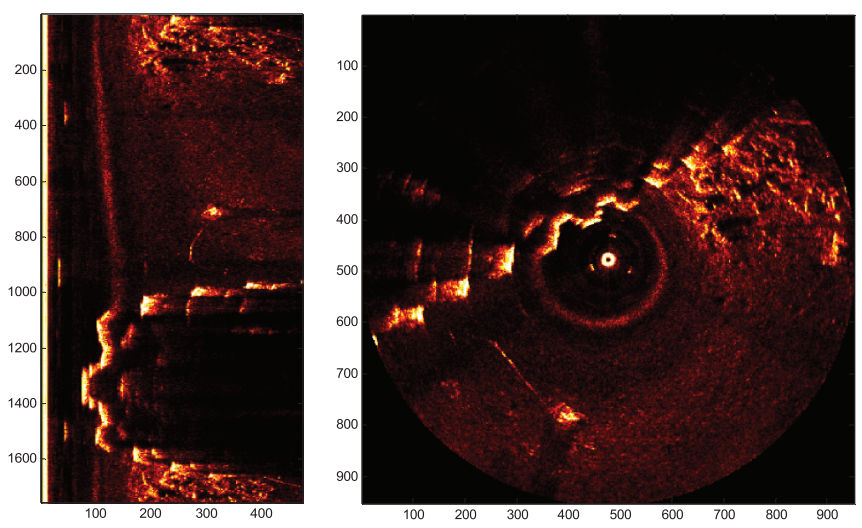

Fig.1.Image representation in polar space (left) and Cartesian space (right)

\section{SIMULATING SONAR IMAGES}

There are a number of documented studies on generation of simulated sonar images based on the known seabed shape [7]. The research studies upon these issues performed at the West Pomeranian University of Technology, Szczecin, resulted in building a complete simulator making use of the raytracing method [8]. Although it was built with the intention to cooperate with SSS images in marine applications, the simulator makes it possible to take into account the model of an arbitrary sonar and arbitrary seabed shape via full implementation of the physical model of the environment and acoustic wave propagation in water.

Table1.Comparing input data for SSS and MSIS image simulators.

\begin{tabular}{|c|c|c|}
\hline \multirow{2}{*}{} & \multicolumn{2}{|c|}{ Input parameters for simulation } \\
\cline { 2 - 4 } & SSS images & MSIS images \\
\hline 1 & $\begin{array}{c}\text { Model of sonar converter } \\
\text { and settings: pulse length, } \\
\text { amplification, etc. }\end{array}$ & $\begin{array}{c}\text { Model of sonar converter } \\
\text { with scanning parameters }\end{array}$ \\
\hline 2 & $\begin{array}{c}\text { Route, speed and inclinations of } \\
\text { the unit }\end{array}$ & $\begin{array}{c}\text { Hypothetical location of the } \\
\text { converter, rotation angle } \varepsilon\end{array}$ \\
\hline 3 & $\begin{array}{c}\text { Layered hydrological model of } \\
\text { water depth }\end{array}$ & $\begin{array}{c}\text { Simplified hydrological } \\
\text { model of water depth }\end{array}$ \\
\hline 4 & $\begin{array}{c}\text { Geometric model of the seabed } \\
\text { with information on surface type }\end{array}$ & $\begin{array}{c}\text { Geometric model of the } \\
\text { seabed in GRID form }\end{array}$ \\
\hline
\end{tabular}

For the purpose of generation of synthetic MSIS images, part of input parameters of the simulation is intentionally omitted or averaged. This mainly refers to parameters describing the motion of the vessel and to the hydrological model of water depth. The route of the vessel is replaced by a point which defines the sought position of the sonar and the attributed course. This course changes after each converter rotation by the angle $\varepsilon$. The environmental conditions in shallow port areas allow to simplify the curvilinear model which relates the speed of sound with the water depth to one value assumed for the entire water depth (the stationary sonar is located on the seabed and works within a small observation range, as a result of which minor speed-of-sound differences 
can be neglected). When the sonar images are recorded in the port area of high salinity, the software used for data acquisition can attach the additional information on the speed-of-sound profile in water, recorded by the SVP (Sound Velocity Profiler) probe, to the raw sonar data. The remaining parameters, such as: frequency of the generated signal, spatial range, amplification, breadth of the beam, and other recording data which are needed to generate the synthetic image, are stored in real sonar data files and can be read each time we want to generate a new series of simulated images.

\section{HIGH-DENSITY BATHYMETRIC DATA}

The sonar search over the seabed is most frequently performed in a water region with known bathymetry. The bathymetric measurements are usually made immediately before sonar measurements, which means that these two databases are relatively consistent in spatio-temporal terms. Due to high changeability of water environment, the above data consistency is very important, as the seabed shape affects directly both types of data. To make the generation of synthetic sonar images possible, an accurate GRID model of seabed is to be acquired in the form of a depth grid with the mesh size close to that of a pixel in the original image.

The numerical model of seabed [9-15] is constructed by interpolating the depth values at the measuring points with the aid of the selected interpolation algorithm. Most usually, the model has the form of a grid of regular squares with depth values attributed to each grid node. The map constructed in this way does not reflect precisely the characteristic of the seabed, nor it allows to identify objects on it; it only represents an approximate shape of the seabed. The accuracy of this approximation depends on the applied technology and physical characteristics of the converter(s) [16].

\section{CONCEPT OF THE POSITIONING METHOD}

Comparative navigation methods consist in comparing different available data sets, some of which have certain spatial references. The general concept of the method assumes that, based on the bathymetric model of the seabed, a synthetic image can be generated for an arbitrary point within the spatial range of this model using the sonar image simulator. The generated images constitute the database of images, which is then used for comparing with real images. Finding the best matching pair of images with the aid of a given similarity function provides the information about the geospatial position of the real image.

\section{PROCESS FLOWCHART}

Figure 2 shows a full scheme of sonar image georeferencing.

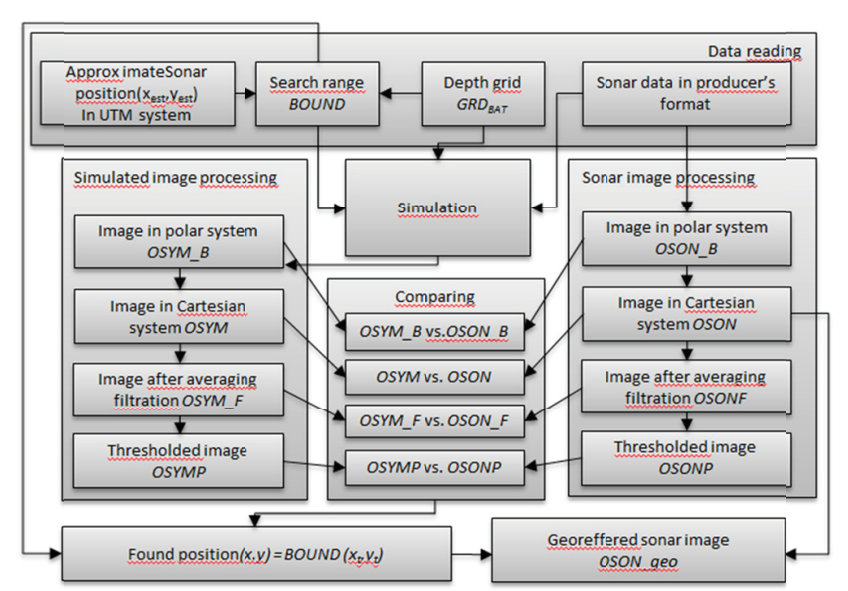

Fig.2. Scheme of finding geographical location of the real image.

The model of seabed $G R D_{B A T}$ and the information on the approximate sonar position $\left(x_{e s t}, y_{\text {est }}\right)$ are used for determining the boundaries for the rectangular area of search BOUND. The images for the area BOUND are generated by the simulator in series. All parameters defined for one series are constant, regardless of the position at which consecutive synthetic images are generated. When starting the georeferencing process, all input data are read and generation of the series of reference images begins. The generator saves the results as images in polar coordinate system. Each subsequent image is simulated based on $G R D_{B A T}$ data with the set position shift $(\Delta x, \Delta y)$ and saved in a separate file. The real image and each simulated image in the series (Fig.3) are subject to: (1) further transformation to the Cartesian space and visualisation using the polar linear interpolation [17] to the OSYM form (Fig.4), and (2) averaging with the aid of the convolution filter with mask $5 \times 5$ (OSYMF form) and thresholding which emphasizes cutting-off of the average values from shadows in the image (OSYMP form) (Fig. 5).
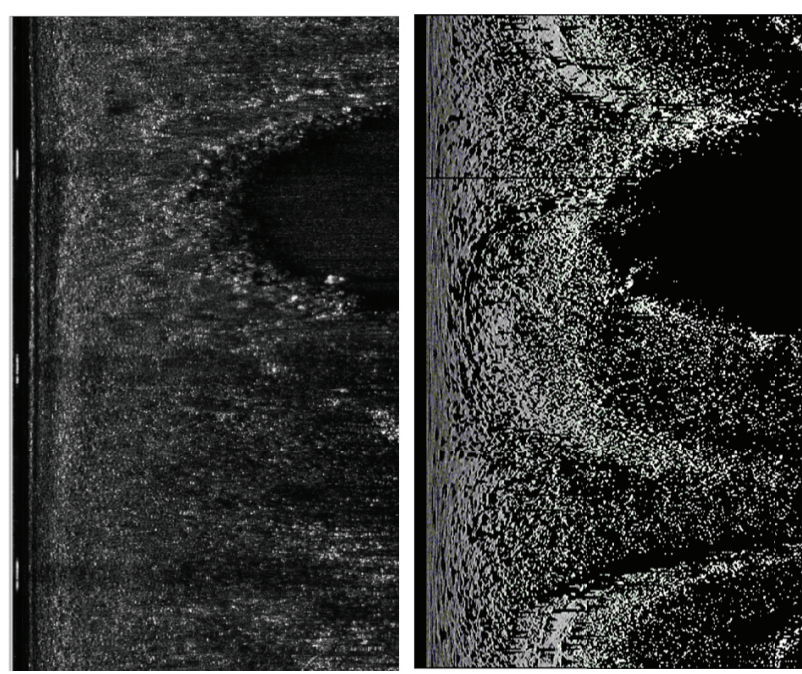

Fig.3. Sample pair of corresponding images OSON_B $B_{T}$ and OSYM_B 


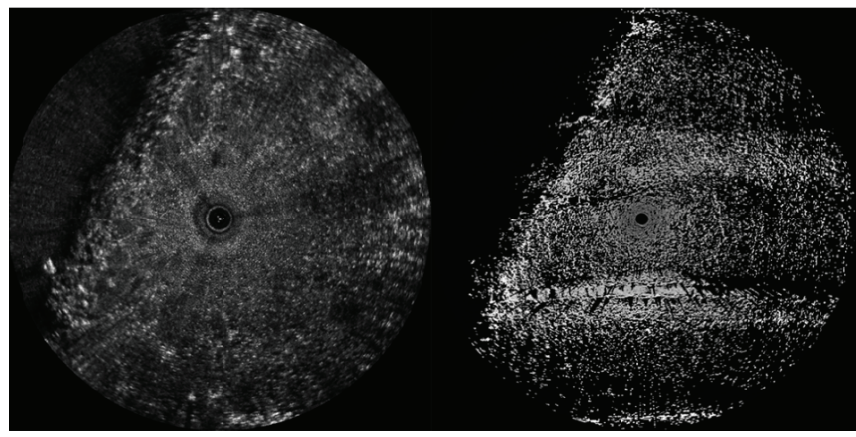

Fig. 4. Sample pair of corresponding images $\mathrm{OSON}_{T}$ and OSYM
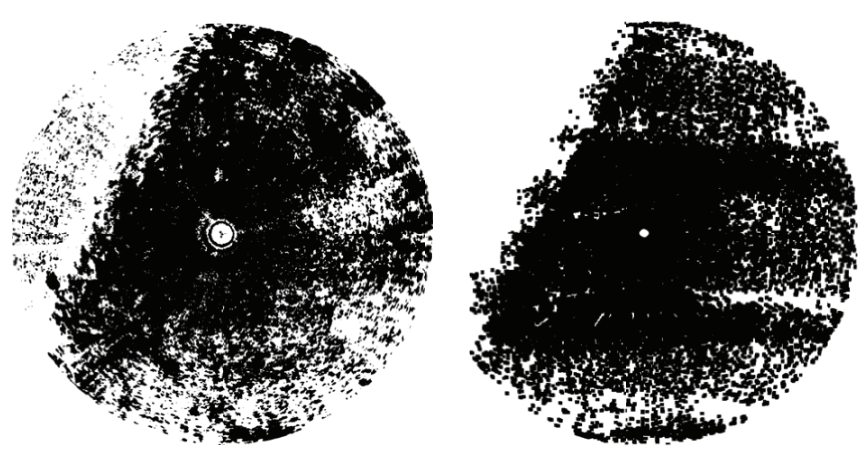

Fig.5 Sample pair of corresponding images OSONP ${ }_{T}$ and $O S Y M P_{T}$

The result of each operation is saved in a separate file. The images prepared in the above way are ready for comparing. Depending on the selected similarity function, relevant transformations of the real image and the simulated image are compared with each other. The simulated image with the highest similarity coefficient is considered the best matching, and the position $T(x B O U N D, y B O U N D)$ for which it was generated indicates the position of the sonar head during real recording. That means that the position of the sonar image matrix centre position has been established and full georeference has been attributed to all pixels of the image. If the value of the selected similarity coefficient is not sufficiently high, the position is considered unknown.

\section{COMPARATIVE NAVIGATION METHODS}

The comparative navigation methods have been mainly used, so far, for positioning with the aid of a radar and electronic navigation maps [18-20], although in recent years the comparison methods are becoming more and more frequently used in underwater positioning [21-26]. The research direction which has recently been very popular in this area refers to mobile navigation [27-29].

There are a number of similarity functions which make it possible to identify relations between images. In those cases, the comparison between the real image and the synthetic image as a whole is done using a method which allows to determine global difference, or global similarity between them. The deterministic methods include comparative navigation, mainly performed using distance and proximity functions, as well as correlation and logical conjunction methods [18]. The research reported in the article focuses on methods generating best results for SSS images and those which after transformation can take into account the specificity of MSIS imaging. The basic method here is the Euclidean distance:

$$
D=\sqrt{\sum_{n}\left(O S O N_{n}-O S Y M_{n}\right)^{2}}
$$

and the closely related basic difference algorithm:

$$
R=\sum_{n} \frac{\mid \operatorname{OSON}_{n}-\mathrm{OSYM}_{n}}{N}
$$

where:

$\mathrm{OSON}_{n}$ and $\mathrm{OSYM}_{n}$ - values of pixels,

$N$ - total number of $n$ pixels in the image.

However, the methods which are most frequently used for comparing images are the correlation methods. They analyse correlations between matrices. The most common is the linear correlation:

$$
\rho_{O S, O W}=\frac{\sum_{n}\left(O S O N-\mu_{O S O N}\right)\left(O S Y M-\mu_{O S Y M}\right)}{\sqrt{\sum_{n}\left(O S O N-\mu_{O S O N}\right)^{2} \sum_{n}\left(O S Y M-\mu_{O S Y M}\right)^{2}}}
$$

where:

$\mu_{O S Y M}, \mu_{O S O N}-$ arithmetic means of image elements.

To make the method insensitive to image rotation, phase correlation can be used after applying the Fourier-Mellin transformation to image invariants. The phase correlation function:

$$
r_{f g(m, n)}=\frac{1}{M N} \sum_{k_{1} k_{2}} R_{F G}\left(k_{1} k_{2}\right) W_{M}^{-k_{1} m} W_{M}^{-k_{2} n}
$$

is the discrete two-dimensional inverse Fourier transform (2D IDFT):

$$
R_{F G}\left(k_{1} k_{2}\right)=\frac{F\left(k_{1} k_{2}\right) G^{*}\left(k_{1} k_{2}\right)}{\left|F\left(k_{1} k_{2}\right) G^{*}\left(k_{1} k_{2}\right)\right|}
$$

where $R_{F G}\left(k_{1} k_{2}\right)$ is the mutual phase spectrum between two discrete Fourier transforms (2D DFT):

$$
\begin{gathered}
F\left(k_{1} k_{2}\right)=\sum_{m, n} f(m, n) W_{M}^{k_{1} m} W_{M}^{k_{2} n} \\
\text { and } \\
G\left(k_{1} k_{2}\right)=\sum_{m, n} g(m, n) W_{M}^{k_{1} m} W_{M}^{k_{2} n}
\end{gathered}
$$

of the analysed images. In formulas (6)-(7):

$f(m, n), g(m, n)$ - the compared images, each of dimensions $M x N$, with the indices ranging within: $m=-M_{2}$.. $M_{2}\left(M_{2}>0\right)$ and $n=-N_{2} \ldots N_{2}\left(N_{2}>0\right)$.

$$
k_{1}=-M_{2} . . M_{2} ; k_{2}=-N_{2} . . N_{2} \text {, }
$$




$$
W_{M}=e^{-j \frac{2 \pi}{M}}, W_{N}=e^{-j \frac{2 \pi}{N}}
$$

The most effective tools in comparative navigation making use of SSS images appear to be the algorithms making use of the logical conjunction method as the similarity function [18]. To adapt this function to the specificity of MSIS images, the areas without sonar information are to be eliminated from calculations, as they would always make good match. The final matching coefficient, taking the values from the interval $<0,1>$ can be described by the formula:

$$
P_{u p r}=\frac{\left(\sum_{k=1}^{K} w s p_{k}\right)-\left(\sum_{k=1}^{K} w s p_{o}\right)}{N-N_{o}}
$$

where:

$w s p_{k}$ - pixel matching coefficient, equal to one for each pair of corresponding pixels when their values in OSON and OSYM images are the same.

$w s p_{0}$ - pixel matching coefficient, equal to zero for each pair of corresponding pixels in the OSON and OSYM areas without sonar information,

$N$, - total number of $n$ pixels in the image,

$N_{o}$ - total number of $n_{0}$ pixels in the image (without sonar information).

\section{EXPERIMENT}

The experiment made use of real MSIS images recorded by sonar MS1000 on the selected area of the Szczecin-Świnoujście port complex. The model of seabed was created using highdensity bathymetric data obtained from the interferometric system GeoSwathPlus for the area of interest. The issues of processing of high-density data obtained using remote sensing methods are discussed in [30-41], among other sources.

Synthetic sonar images were generated using a simulator built at the West Pomeranian University of Technology as part of PhD research [8]. After collecting all needed data, the database of synthetic images was created.

\section{EXAMINING COMPARISON METHODS}

As the first stage of experiment, comparison was made between all comparison methods mentioned in the previous section. It consisted in comparing four real test images with 22-30 sets of simulated images (along with their transformations), including those generated from correct position. Depending on the selected comparison method, relevant pairs of transformations of corresponding images were compared. The basic criterion when evaluating the correctness of image finding was the value of the matching coefficient $\mathrm{F}$ which determined the degree of similarity. Selected sample results for this comparison series are collated in Table 2.
Table2. Sample results of comparison of the real sonar image with a series of generated synthetic images. Comparison made using different

\begin{tabular}{|c|c|c|c|c|c|}
\hline \multirow{2}{*}{ Item } & \multicolumn{5}{|c|}{ Similarity function value (F) } \\
\hline & $\begin{array}{c}\text { Linear } \\
\text { correlation }\end{array}$ & $\begin{array}{c}\text { Logical } \\
\text { conjunction }\end{array}$ & $\begin{array}{c}\text { Euclidean } \\
\text { distance }\end{array}$ & $\begin{array}{l}\text { Difference } \\
\text { algorithm }\end{array}$ & $\begin{array}{c}\text { Phase } \\
\text { correlation }\end{array}$ \\
\hline 1 & 0.354694 & 0.608413 & 89723 & 99.854805 & 0.845871 \\
\hline 2 & 0.503885 & 0.642764 & 79685 & 91.09513 & 0.85291 \\
\hline 3 & 0.535903 & 0.633744 & 70318 & 93.395388 & 0.827095 \\
\hline 4 & 0.589224 & 0.637099 & 68991 & 92.539793 & 0.824445 \\
\hline 5 & 0.522007 & 0.714866 & 65595 & 72.709124 & 0.808766 \\
\hline 6 & 0.450099 & 0.634971 & 85592 & 93.082478 & 0.855178 \\
\hline 7 & 0.438632 & 0.647589 & 66355 & 89.864901 & 0.78797 \\
\hline 8 & 0.523224 & 0.62137 & 65531 & 96.550526 & 0.787347 \\
\hline 9 & 0.551668 & 0.654039 & 70826 & 88.220075 & 0.805705 \\
\hline 10 & 0.430721 & 0.604436 & 56726 & 100.86882 & 0.793401 \\
\hline 11 & 0.597485 & 0.608913 & 63594 & 99.727073 & 0.780116 \\
\hline 12 & 0.43882 & 0.614555 & 97864 & 98.288475 & 0.891996 \\
\hline 13 & 0.512393 & 0.57534 & 85751 & 108.28837 & 0.876169 \\
\hline 14 & 0.480533 & 0.550385 & 61188 & 114.65181 & 0.807209 \\
\hline 15 & 0.485056 & 0.603464 & 82092 & 101.11679 & 0.797731 \\
\hline 16 & 0.509599 & 0.674935 & 75940 & 82.891698 & 0.785828 \\
\hline 17 & 0.574519 & 0.656217 & 78636 & 87.664545 & 0.790364 \\
\hline 18 & 0.557915 & 0.666906 & 78698 & 84.938988 & 0.79083 \\
\hline 19 & 0.633638 & 0.712073 & 73344 & 73.421287 & 0.786941 \\
\hline 20 & 0.685932 & 0.749959 & 70092 & 63.760347 & 0.78894 \\
\hline 21 & 0.57764 & 0.684403 & 78292 & 80.477266 & 0.793146 \\
\hline 22 & 0.483482 & 0.709421 & 67611 & 74.097772 & 0.763108 \\
\hline 23 & 0.580108 & 0.699905 & 68792 & 76.524333 & 0.768687 \\
\hline
\end{tabular}
similarity functions.

In the presented series the sample No. 20 was the image generated from the real sonar position - TH $(0,0)$. Three methods indicated this sample as the image matching position. The phase correlation method, which is believed to be most promising for circular MSIS images, did not provide expected results. This method operates on images in the form of invariants, i.e. images transformed to the polar coordinate system (Fig. 3).

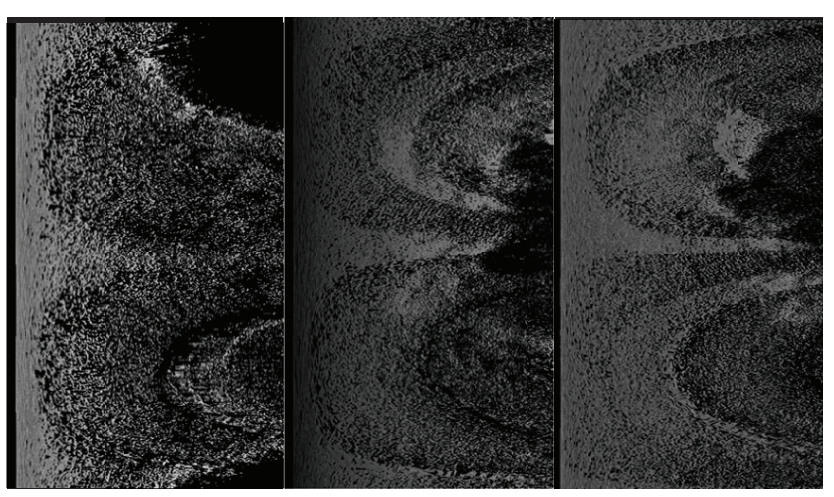

Fig. 6. Original image rotated in polar system (left) and two samples with the highest phase correlation coefficients (right). 
Theoretically, along with eliminating the image rotation error, (Fig. 6) it can indicate a possible image shift direction. Unfortunately, its use for identification purposes in the reported tests failed in all examined test cases, which could be an effect of the nature of the bathymetric test data used for image simulation. Local data errors resulting from the applied acquisition method affected the quality of the generated synthetic image by generating gentle linear changes in the image, which in turn affected considerably the calculated phase correlation coefficient.

Due to excessively large differences in values of corresponding image pixels, the simplest method, i.e. the Euclidean distance method turned out useless for georeferencing of both OSON and OSYM images, and their filtered versions. On the other hand, the difference method, used after image binarization, allowed to identify correct positions in $77 \%$ of test images. The linear correlation and logical conjunction methods returned the best results. Each time they correctly selected from the series the synthetic image which corresponded to the selected real image. The correlation was performed for pairs of OSON and OSYM images, and their OSONP and OSYMP versions obtained after thresholding.

\section{LINEAR CORRELATION AND LOGICAL CONJUNCTION METHODS}

As the further step of examination, matching coefficients were checked for the linear correlation method and the logical conjunction method. The RMSD (Root Mean Square Deviation) error was also calculated:

$$
\sigma(F)=\sqrt{\frac{\sum_{n=1}^{N}\left(F_{n}-F_{S r}\right)^{2}}{N-1}}
$$

where:

$F_{n} \quad-$ matching coefficient value for n-th comparison,

$F_{s r} \quad$ - matching coefficient average,

$N \quad$ - number of comparisons.

The matching coefficient average was much higher for the logical conjunction method (Table 3). Low value of the RMSD error confirmed correct selection of methods for finding corresponding pairs of images.

Table3. Comparing correlation and logical conjunction methods, with selected matching function values for corresponding pairs of images.

\begin{tabular}{|c|c|c|c|}
\hline \multirow{2}{*}{ Item } & \multicolumn{3}{|c|}{$F$} \\
\cline { 2 - 4 } & $\begin{array}{c}\text { Linear } \\
\text { correlation }\end{array}$ & $\begin{array}{c}\text { Linear } \\
\text { correlation } \\
\text { (thresh.) }\end{array}$ & $\begin{array}{c}\text { Logical } \\
\text { conjunction }\end{array}$ \\
\hline $\boldsymbol{1}$ & 0.729991 & 0.744989 & 0.841871 \\
\hline $\mathbf{2}$ & 0.685932 & 0.608394 & 0.749959 \\
\hline $\mathbf{3}$ & 0.78813 & 0.73441 & 0.82319 \\
\hline 4 & 0.64534 & 0.67392 & 0.79147 \\
\hline $\mathbf{5}$ & 0.73458 & 0.79421 & 0.87519 \\
\hline $\boldsymbol{F}_{s r}$ & $\mathbf{0 . 7 1}$ & $\mathbf{0 . 7 1}$ & $\mathbf{0 . 8 3}$ \\
\hline $\boldsymbol{\sigma}(\boldsymbol{F})$ & $\mathbf{0 . 0 5 4}$ & $\mathbf{0 . 0 7 1}$ & $\mathbf{0 . 0 4 7}$ \\
\hline
\end{tabular}

Additionally, tests were performed for a series of images generated in direct vicinity of the real image position $\mathrm{TH}(0,0)$ (Table 4). The image generation position was gradually shifted by the multiples of $0,5 \mathrm{~m}$ in 8 directions, up to the maximal distance of $2,5 \mathrm{~m}$. The matching function values decreased with the increasing shift from the initial position $T H(0,0)$.

Table4. Comparing the correlation and logical conjunction methods via comparing pairs of images shifted with respect to each other.

\begin{tabular}{|c|c|c|c|c|}
\hline \multirow{2}{*}{ Item } & \multirow{2}{*}{$\begin{array}{c}\text { Shift } \\
\text { [m] }\end{array}$} & $\begin{array}{c}\text { Linear } \\
\text { correlation }\end{array}$ & $\begin{array}{c}\text { Linear } \\
\text { correlation } \\
\text { (thresh.) }\end{array}$ & $\begin{array}{c}\text { Logical } \\
\text { conjunction }\end{array}$ \\
\hline $\mathbf{1}$ & 0.5 & 0.597820 & 0.726448 & 0.830210 \\
\hline $\mathbf{2}$ & 1 & 0.643427 & 0.701464 & 0.814532 \\
\hline $\mathbf{3}$ & 2.5 & 0.541339 & 0.513479 & 0.699780 \\
\hline $\mathbf{4}$ & 0.5 & 0.695637 & 0.734348 & 0.835071 \\
\hline $\mathbf{5}$ & 1 & 0.677591 & 0.713156 & 0.821616 \\
\hline $\mathbf{6}$ & 5 & 0.614527 & 0.616787 & 0.759780 \\
\hline $\mathbf{7}$ & 0 & 0.729991 & 0.744989 & 0.841871 \\
\hline
\end{tabular}

Finally, the two methods were tested for possible introduction of a real sonar image which did not come from the area defined by the matrix BOUND. Selected sample results are given in Table 5 .

Table 5. Sample results of matching of the real sonar image which was not recorded in the test area with the series of generated samples.

\begin{tabular}{|c|c|c|c|}
\hline \multirow[b]{2}{*}{ Item } & \multicolumn{3}{|c|}{$F$} \\
\hline & $\begin{array}{c}\text { Linear } \\
\text { correlation }\end{array}$ & $\begin{array}{c}\text { Linear } \\
\text { correlation } \\
\text { (thresh.) }\end{array}$ & $\begin{array}{c}\text { Logical } \\
\text { conjunction }\end{array}$ \\
\hline 1 & 0.373771 & 0.277592 & 0.446179 \\
\hline 2 & 0.322291 & 0.182071 & 0.508113 \\
\hline 3 & 0.416403 & 0.29997 & 0.459867 \\
\hline 4 & 0.422898 & 0.282476 & 0.429769 \\
\hline 5 & 0.389624 & 0.225019 & 0.420389 \\
\hline 6 & 0.362077 & 0.185763 & 0.40462 \\
\hline 7 & 0.296578 & 0.08056 & 0.366817 \\
\hline 8 & 0.257341 & 0.194632 & 0.275026 \\
\hline 9 & 0.348041 & 0.228477 & 0.309079 \\
\hline 10 & 0.328448 & 0.152326 & 0.343959 \\
\hline 11 & 0.431186 & 0.257881 & 0.433348 \\
\hline 12 & 0.369198 & 0.245872 & 0.415836 \\
\hline
\end{tabular}




\begin{tabular}{|c|c|c|c|}
\hline 13 & 0.328325 & 0.233356 & 0.273214 \\
\hline 14 & 0.251602 & 0.130849 & 0.380071 \\
\hline 15 & 0.360699 & 0.142585 & 0.33828 \\
\hline 16 & 0.404924 & 0.205724 & 0.348561 \\
\hline 17 & 0.229483 & 0.043314 & 0.489395 \\
\hline 18 & 0.409045 & 0.140118 & 0.360576 \\
\hline 19 & 0.317168 & 0.203989 & 0.252424 \\
\hline 20 & 0.29442 & -0.015052 & 0.214406 \\
\hline 21 & 0.121923 & -0.21945 & 0.300226 \\
\hline 22 & 0.36964 & 0.217668 & 0.257096 \\
\hline 23 & 0.36904 & 0.19259 & 0.386883 \\
\hline 24 & 0.423177 & 0.216471 & 0.358698 \\
\hline 25 & 0.419089 & 0.238363 & 0.37089 \\
\hline 26 & 0.434638 & 0.20227 & 0.413055 \\
\hline 27 & 0.451125 & 0.21614 & 0.450957 \\
\hline 28 & 0.433396 & 0.247289 & 0.382619 \\
\hline 29 & 0.348118 & 0.249353 & 0.488499 \\
\hline 30 & 0.409815 & 0.230515 & 0.467359 \\
\hline
\end{tabular}

In this final test the values of the matching coefficients for the two examined methods turned out low. For the correlation method they did not exceed 0,5 which is interpreted as indication that, according to this method, none of the analysed image pairs are similar to each other, which is true. Also the logical conjunction value is not satisfactorily high to identify the image position. Thus the final limit of the method can be formulated which says that the image position can be identified provided that certain value of the similarity coefficient is reached.

\section{CONCLUSIONS}

The results of the performed research allow to conclude that the linear correlation method and the logical conjunction method are most useful for finding similarities between the images obtained from stationary scanning sonars. This conclusion is in line with that resulting from their common application for radar image based comparative navigation. The obtained results have revealed that the similarity function which returns best matching is the logical conjunction method, adapted to the specificity of MSIS images. The threshold value of the matching coefficient which allowed to decide whether the similarity is sufficiently high for assuming the hypothetical position $T$ as the sonar position $T H(0,0)$, was assumed at the level of 0,75 .

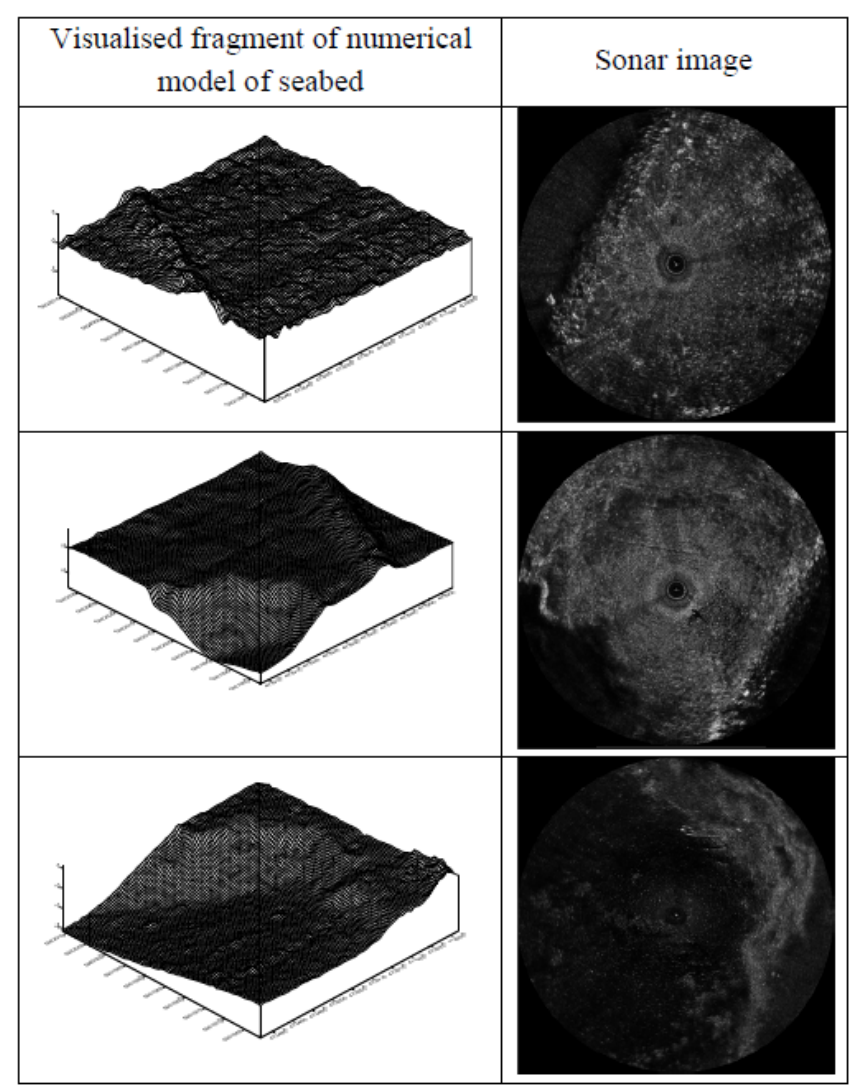

Fig.7. Numerical models of seabed fragments corresponding to selected georeferred test images.

The proposed positioning solution, which bases on the above comparison methods, is part of wider research performed by the authors upon improving the interpretation potential of sonar images. Precise data georeferencing allows to link these data with other pieces of information, which can be attached as successive channels of the sonar image. Additionally, the developed method is of great practical importance in supporting the process of high-resolution imaging of underwater port areas with the aid of the stationary MSIS.

The implemented stand-alone module for comparing real images with the database of synthetic images allows to analyse different similarity functions for different forms of input data, which is of certain importance for future tests of the method, to be performed on the data coming from other water regions with different seabed types.

The method presented in the article was the essence of the patent application submitted by the authors [42]. 


\section{BIBLIOGRAPHY}

1. Wawrzyniak N., Zaniewicz G., Detecting small moving underwater objects using scanning sonar in waterside surveillance and complex security solutions. Proceedings of the 17th International Radar Symposium (IRS),Krakow, Poland,(2016)

2. Ribas D., Ridao P., Neira J.et al. Line extraction from mechanically scanned imaging sonar, 3rd Iberian Conference on Pattern Recognition and Image Analysis. Book Series: Lecture Notes in Computer Science, vol.4477, pp. 322-329, (2007).

3. Kazimierski, W., Zaniewicz, G., Analysis of the Possibility of Using Radar Tracking Method Based on GRNN for Processing Sonar Spatial Data, Proceedings of the Joint Rough Set Symposium, Spain, Kryszkiewicz et al. (Eds), Lecture Notes in Artificial Intelligence, 8537, pp. 319-326. Granada and Madrid, (2014).

4. Zhang, J., Han, Y., Zheng, C. et al., Underwater target localization using long baseline positioning system. Applied Acoustics, vol. 111, pp. 129-134, (2016).

5. Reis, J., Morgado, M., Batista, P. et al., Design and Experimental Validation of a USBL Underwater Acoustic Positioning System. Sensors, vol. 16, issue 9, article number 1491, (2016).

6. Naus, K., Nowak, A., The Positioning Accuracy of BAUV Using Fusion of Data from USBL System and Movement Parameters Measurements. Sensors, vol. 16, issue 8, article number 1279, (2016)

7. Bell, J. M., Linnett L. M., Simulation and Analysis of Synthetic Sidescan Sonar Images. IEEE Proceedings Radar, Sonar and Navigation, 144(4), (1997).

8. Palczynski, M., Method for generating synthetic sonar images for the purpose of comparative navigation, (Ph. D. thesis), Szczecin University of Technology, (2008).

9. Stateczny, A., The neural method of sea bottom shape modelling for the spatial maritime information system. Book Editor(s): Brebbia, CA., Olivella, J. Maritime Engineering and Ports II. Book Series: Water Studies Series vol. 9, pp. 251-259, Barcelona (2000).

10. Lubczonek, J., Stateczny, A., Concept of neural model of the sea bottom surface. Book Editor(s): Rutkowski, L., Kacprzyk, J. Neural Networks and Soft Computing, Book Series: Advances in Soft Computing, pp. 861-866, Zakopane (2003).

11. Lubczonek, J., Hybrid neural model of the sea bottom surface, Edited by: Rutkowski, L., Siekmann, J.,
Tadeusiewicz, R. et al., 7th International Conference on Artificial Intelligence and Soft Computing, Lecture Notes in Artificial Intelligence, vol. 3070, pp. 1154-1160, Zakopane, Poland (2004).

12. Maleika, W., Moving Average Optimization in Digital Terrain Model Generation Based on Test Multibeam Echosounder Data, Geo-Marine Letters, 35, 61-68, (2015).

13. Maleika, W., The Influence of the Grid Resolution on the Accuracy of the Digital Terrain Model Used in Seabed Modelling. Marine Geophysical Research, 36, 35-44, (2015).

14. Maleika, W, Palczynski, M., Frejlichowski, D., Effect of Density of Measurement Points Collected from a Multibeam Echosounder on the Accuracy of a Digital Terrain Model, 4th International Scientific Asian Conference on Intelligent Information and Database Systems (ACIIDS), Edited by: Pan, JS., Chen, SM., Nguyen, NT., Book Series: Lecture Notes in Artificial Intelligence, vol. 7198, pp. 456-465, Kaohsiung, Taiwan, (2012).

15. Maleika, W., The influence of track configuration and multibeam echosounder parameters on the accuracy of seabed DTMs obtained in shallow water, Earth Science Informatics, vol. 6, issue 2, pp. 47-69, (2013).

16. Wawrzyniak, N., Hyla, T., Managing Depth Information Uncertainty in Inland Mobile Navigation Systems. Joint Rough Set Symposium, Granada and Madrid, Spain, Kryszkiewicz et al. (Eds), Lecture Notes in Artificial Intelligence, 8537, pp. 343-350, 2014.

17. Ratuszniak, N., Palczynski, M., Method of visualization for scanning sonar image, Measurement Automation and Monitoring vol.56 no 12/2010.

18. Stateczny, A., Methods of comparative plotting of the ship's position. Book Editor(s): Brebbia, CA., Sciutto, G. Maritime Engineering \& Ports III. Book Series: Water Studies Series vol. 12, pp. 61-68, Rhodes (2002)

19. Stateczny, A., Artificial neural networks for comparative navigation. Book Editor(s): Rutkowski, L., Siekmann, J., Tadeusiewicz, R., et al. Artificial Intelligence and Soft Computing - ICAISC 2004. Book Series: Lecture Notes in Artificial Intelligence, vol. 3070, pp. 1187-1192, Zakopane (2004).

20. Kazimierski, W., Stateczny, A., Radar and Automatic Identification System track fusion in an Electronic Chart Display and Information System. Journal of Navigation, vol. 68, pp 1141-1154, 2015.

21. Wang, L., Yu, L., Zhu, Y., Construction Method of the Topographical Features Model for Underwater Terrain Navigation. Polish Maritime Research, vol. 22, special issue 
1, pp. 121-125, (2015).

22. Zhou, L., Cheng, X., Zhu, Y.,Terrain aided navigation for autonomous underwater vehicles with coarse maps. Measurement Science and Technology, vol. 27, issue 9, article number 095002, (2016).

23. Miller, P., A., Farrell, J., A., Zhao, Y. et al. Autonomous Underwater Vehicle Navigation, IEEE Journal of Oceanic Engineering, vol. 35, issue 3, special issue, pp. 663-678, (2010).

24. Nygren, I., Jansson, M., Terrain navigation for underwater vehicles using the correlator method. IEEE Journal of Oceanic Engineering vol. 29, issue 3, pp. 906-915, (2004).

25. Zhou, L., Cheng, X., Zhu, Y. et al. Terrain Aided Navigation for Long-Range AUVs Using a New Bathymetric Contour Matching Method. IEEE/ASME International Conference on Advanced Intelligent Mechatronics (AIM), Busan, South Korea, pp. 249-254, (2015).

26. Ramesh, R., Jyothi, V., Vedachalam, N. et al. Development and Performance Validation of a Navigation System for an Underwater Vehicle. Journal of Navigation, vol. 69, issue 5, pp. 1097-1113, (2016).

27. Hyla, T., Kazimierski, W., Wawrzyniak, N,. Analysis of Radar Integration Possibilities in Inland Mobile Navigation. Proceedings of 16th International Radar Symposium (IRS), International Radar Symposium Proceedings, H. Rohling (Ed.), pp. 864-869, Dresden, Germany (2015).

28. Hyla, T., Wawrzyniak, N., Kazimierski, W., Model of Collaborative Data Exchange for Inland Mobile Navigation, Proceedings of Soft Computing in Computer and Information Science Conference, Advances in Intelligent Systems and Computing, vol. 342, pp. 435-444, Miedzyzdroje, (2015)

29. Gotlib, D., A Cartographic Presentation Model for Navigation and Location-Based Applications. Joint Symposium of ISPRS Commission IV / AutoCarto Annual Conference. Book Series: International Archives of the Photogrammetry Remote Sensing and Spatial Information Sciences, vol. 38, part 4, Orlando, (2010).

30. Wlodarczyk-Sielicka, M., Stateczny, A., Selection of SOM Parameters for the Needs of Clusterisation of Data Obtained by Interferometric Methods. Proceedings of 16th International Radar Symposium (IRS), International Radar Symposium Proceedings, H. Rohling (Ed.), pp. 1129-1134, Dresden, Germany (2015).

31. Wlodarczyk-Sielicka M., Lubczonek J., Stateczny A., Comparison of Selected Clustering Algorithms of Raw Data Obtained by Interferometric Methods Using Artificial
Neural Networks. Proceedings of 16th International Radar Symposium (IRS), International Radar Symposium, Krakow, Poland (2016).

32. Wlodarczyk-Sielicka M., Stateczny A., Comparison of selected reduction methods of bathymetric data obtained by multibeam echosounder. Proceedings of Baltic Geodesy Congress, Gdansk, Poland (2016).

33. Dziubich, T., Szymanski, J., Brzeski, A. et al.Depth Images Filtering in Distributed Streaming. Polish Maritime Research, vol. 23, issue: 2, pp. 91-98, (2016).

34. Wlodarczyk-Sielicka, M., Stateczny, A., Clustering Bathymetric Data for Electronic Navigational Charts. The Journal of Navigation vol. 69, issue 5, pp 1143-1153 (2016).

35. Hejmanowska B., Kamiński W., Przyborski M., Pyrchla J., Modern remote sensing and the challenges facing education systems in terms of its teaching, 7th International Confrence on Education and New Learning Technologies EDULEARN 15 Barcelona, Spain, Book Series: EDULEARN Proceedings, pp. 6549-6558,(2015).

36. Kazimierski, W., Wlodarczyk-Sielicka, M., Technology of Spatial Data Geometrical Simplification in Maritime Mobile Information System for Coastal Waters. Polish Maritime Research,Vol. 23, Issue:3, pp. 3-12, (2016).

37. Moszynski, M., Chybicki, A., Kulawiak, M. et al. A novel method for archiving multibeam sonar data with emphasis on efficient record size reduction and storage. Polish Maritime Research, vol. 20, issue 1, pp. 77-86, (2013).

38. Stateczny, A., Bodus-Olkowska I., Hierarchical Hydrographic Data Fusion or Precise Port Electronic Navigational Chart Production. in Mikulsk J.(ed.) Telematics in the Transport Environment, Book Series: Communications in Computer and Information Science 471, pp. 359-368, Ustron, Poland (2014).

39. Stateczny, A., Bodus-Olkowska, I., Sensor Data Fusion Techniques for Environment Modelling. Proceedings of 16th International Radar Symposium (IRS), International Radar Symposium Proceedings, H. Rohling (Ed.), pp. 11231128, Dresden, Germany (2015).

40. Pokonieczny, K., Bielecka, E., Kaminski, P., Analysis of Spatial Distribution of Geodetic Control Points and Land Cover. 14th International Multidisciplinary Scientific Geoconference (SGEM) Geoconference on Informatics, Geoinformatics and Remote Sensing, vol. II, pp. 49-56, Albena, Bulgaria, (2014).

41. Burdziakowski, P., Janowski A., Kholodkow A. et al., Maritime Laser Scanning as the Source For Spatial Data, Polish Maritime Research, vol. 22, issue 4, pp. 9-14, (2015). 
42. Stateczny, A., Wawrzyniak, N., Method for determining stationary position of scanning sonar, involves determining head position based on sonar search for actual sonar image with set of synthetic images generated by performing ray tracing process based on model of bottom. Patent Number: PL406523-A1. Patent Assignee: Marine Technology Sp. z o.o. (2015).

\section{CONTACT WITH THE AUTHOR}

Natalia Wawrzyniak

e-mail:n.wawrzyniak@am.szczecin.pl

Maritime University

Szczecin

Poland 